\title{
FINDING INCOMES OF THE HM-NETWORK WITH ONE-TYPE MESSAGES BYPASS OF SYSTEMS
}

\author{
Mikhail Matalytski ${ }^{1}$, Victor Naumenko ${ }^{2}$ \\ ${ }^{I}$ Institute of Mathematics, Czestochowa University of Technology \\ Czestochowa, Poland \\ ${ }^{2}$ Faculty of Mathematics and Computer Science, Grodno State University \\ Grodno, Belarus \\ m.matalytski@gmail.com,victornn86@gmail.com
}

\begin{abstract}
The object of research is an open HM-network with one-type messages bypass of systems in the transient behavior. Two cases are considered: when incomes from transitions between network states are deterministic functions depending on states and time, and network systems are single-line, and when incomes from transitions between network states are functions depending on random variables. The purpose of the research is to find the expected incomes of such a network in both cases on condition that the probabilities of messages bypasses of systems network and the parameters of incoming flow of messages and services depend on time. Examples are considered.
\end{abstract}

\section{General information}

In [1], a study was conducted in open exponential networks with multiline queuing systems (QS) with one-type messages bypass of systems in transient behavior. In [2] we found the nonstationary state probabilities and the average characteristics of the network in the case, when the probabilities of messages bypasses of systems network and parameters of incoming flow of messages and services depend on time.

In this paper there are considered open HM (Howard-Matalytski) - network incomes of such type, when the probabilities of messages bypasses of systems network depend on time. Here we will consider two cases: when incomes from transitions between network states are deterministic functions depending on states and time and the QS networks are single-line, and when incomes from transitions between network states are functions depending on the random variables (RV).

\section{Formulation of the problem}

Consider an open exponential QN with one-type messages, consisting of $n$ QS $S_{1}, S_{2}, \ldots, S_{n}$. Messages during the transition from one to another QS bring some 
income and, accordingly, the income of the first system is reduced by that amount. It is necessary to find the expected incomes systems in the network during the time $t$ o condition that we know its state at the initial time $t_{0}$.

Let $m_{i}$ be the number of identical service lines in the QS $S_{i}, I_{i}$ - a vector of dimension $n$, consisting of zeros except the $i$-th component, which is equal to 1 , $i=\overline{1, n} ; p_{i j}$ - the transition probability of the message after service in the system $S_{i}$ into the system $S_{j}, i, j=\overline{0, n}$. We assume that the system $S_{0}$ is the external environment. Let us consider the case when the parameters of the incoming flow of messages and services depend on time, i.e. the time interval $[t, t+\Delta t)$ in the network receives a message with a probability $\lambda(t) \Delta t+o(\Delta t)$, and if at the time $t$ of service on the line $i$-th QS located in a message, at the range $[t, t+\Delta t)$ of its services will end with a probability $\mu_{i}(t) \Delta t+o(\Delta t), i=\overline{1, n}$. The message is sent to the $i$-th QS with probability $p_{0 i}, \sum_{i=1}^{n} p_{0 i}=1$. The message sent to this QS from the external environment at a moment of time $t$, with a probability $f^{(i)}(k, t)$ when the network is in a state $(k, t)$, joins the queue, and the probability $1-f^{(i)}(k, t)$ is not attached to the queue, regardless of the handled (i.e., its time of service with a probability of 1 is equal to zero). If the message has been served in the $i$-th QS, it is likely to be sent immediately to the $j$-th QS with probability $p_{i j}$, and leaves the QN with the probability $p_{i 0}, \sum_{j=0}^{n} p_{i j}=1, i=1, \ldots, n$.

Let $k(t)=(k, t)=\left(k_{1}, k_{2}, \ldots, k_{n}, t\right)$ be the state vector of the network, where $k_{i}$ the number of messages at the moment $t$ in the system $S_{i}, i=\overline{1, n} ; \varphi_{i}(k, t)$ - the conditional probability that the message is delivered to the $i$-th QS at time $t$, when the network is in a state $(k, t)$, will not be serviced by any of the QS; $\psi_{i j}(k, t)$ - the conditional probability that the message is delivered to the $i$-th QS outside at time $t$, when the network is in state $(k, t)$, the first time, a service in $j$-th QS; $\alpha_{i}(k, t)$ the conditional probability that the message, served in the $i$-th queuing system at time $t$, when the network is in a state $(k, t)$, will no longer be served in any of QS; $\beta_{i j}(k, t)$ - the conditional probability that the message, served in the $i$-th queuing system at time $t$, when the network is in state $(k, t)$ for the first time then receives services in the $j$-th QS, $i, j=\overline{1, n}$. 


\section{Finding the expected incomes, when incomes from transitions between network states are deterministic functions, depend on states and time}

Let $v_{i}(k, t)$ - total expected income, which the system $S_{i}$ gets during time $t$, if at the initial moment the network is in state $k$, and it is assumed that this function is differentiable in $t ; r_{i}(k)$ - income of system $S_{i}$ at a time when the network is in state $k ; r_{0 i}\left(k+I_{i}, t\right)$ - income of system $S_{i}$, when the network makes a transition from state $(k, t)$ in the state $\left(k+I_{i}, t+\Delta t\right)$ during time $\Delta t ;-R_{i 0}\left(k-I_{i}, t\right)$ - system income, if the network makes a transition from state $(k, t)$ in the state $\left(k-I_{i}, t+\Delta t\right)$; $r_{i j}\left(k+I_{i}-I_{j}, t\right)$ - income of system $S_{i}$ (consumption or loss of system $S_{j}$ ), when the network changes its state from $(k, t)$ to $\left(k+I_{i}-I_{j}, t+\Delta t\right)$ during time $\Delta t$, $i, j=\overline{1, n}$.

Suppose that the network is in state $(k, t)$. During an interval of time it can remain in state $k$ or move to states $\left(k-I_{i}\right),\left(k+I_{i}\right),\left(k+I_{i}-I_{j}\right), i, j=\overline{1, n}$. If the network is in state $(k, t+\Delta t)$, then the expected income of system $S_{i}$ will be $r_{i}(k) \Delta t$ plus the expected income $v_{i}(k, t)$, that it will receive the remaining time $t$ units. The probability of this event is $1-\sum_{i=1}^{n}\left(\lambda(t) p_{0 i}\left(1-\varphi_{i}(k, t)\right)+\mu_{i}(t)\left(1-\beta_{i i}(k, t)\right)\right) \Delta t+o(\Delta t)$. If the network goes to the state $\left(k+I_{i}, t+\Delta t\right)$ with probability $\lambda(t) p_{0 i} \psi_{i j}\left(k+I_{i}, t\right) \Delta t+$ $+o(\Delta t)$, then income of system $S_{i}$ is $\left[r_{0 i}\left(k+I_{i}, t\right)+v_{i}\left(k+I_{i}, t\right)\right]$, and if in state $\left(k-I_{i}, t+\Delta t\right)$ with probability $\mu_{i}(t) \alpha_{i}\left(k-I_{i}, t\right) u\left(k_{i}\right) \Delta t+o(\Delta t)$, then income of the system is $\left[-R_{i 0}\left(k-I_{i}, t\right)+v_{i}\left(k-I_{i}, t\right)\right], i=\overline{1, n}$. Similarly, if the network goes from $(k, t)$ to state $\left(k+I_{i}-I_{j}, t+\Delta t\right)$ with probability $\mu_{j}(t) \beta_{j i}\left(k+I_{i}-I_{j}, t\right) u\left(k_{j}\right) \Delta t+o(\Delta t)$, it brings the system $S_{i}$ income of $r_{i j}\left(k+I_{i}-I_{j}, t\right)$ plus the expected net income for the remaining time, if the initial state of the network was the state $\left(k+I_{i}-I_{j}\right)$. Described above are summarized in Table 1.

Then, using the total probability formula for the mean value of the expected income of the system $S_{i}$ a system of difference-differential equations (DDE) can be obtained:

$$
\begin{gathered}
\frac{d v_{i}(k, t)}{d t}=-\sum_{i=1}^{n}\left[\lambda(t) p_{0 i}\left(1-\varphi_{i}(k, t)\right)+\mu_{i}(t)\left(1-\beta_{i i}(k, t)\right)\right] v_{i}(k, t)+ \\
+\sum_{j=1}^{n}\left[\lambda(t) p_{0 j} \psi_{i j}\left(k+I_{j}, t\right) v_{i}\left(k+I_{j}, t\right)+\mu_{j}(t) \alpha_{j}\left(k-I_{j}, t\right) u\left(k_{j}\right) v_{i}\left(k-I_{j}, t\right)\right]+
\end{gathered}
$$




$$
\begin{aligned}
&+ \sum_{\substack{j=1 \\
j \neq i}}^{n}\left[\mu_{j}(t) \beta_{j i}\left(k+I_{i}-I_{j}, t\right) u\left(k_{j}\right) v_{i}\left(k+I_{i}-I_{j}, t\right)+\right. \\
&\left.+\mu_{i}(t) \beta_{i j}\left(k-I_{i}+I_{j}, t\right) u\left(k_{i}\right) v_{i}\left(k-I_{i}+I_{j}, t\right)\right]+ \\
&+ \sum_{\substack{j=1 \\
j \neq i}}^{n}\left[\mu_{j}(t) \beta_{j i}\left(k+I_{i}-I_{j}, t\right) u\left(k_{j}\right) r_{i j}\left(k+I_{i}-I_{j}, t\right)+\right. \\
&\left.+\mu_{i}(t) \beta_{i j}\left(k-I_{i}+I_{j}, t\right) u\left(k_{i}\right) r_{j i}\left(k-I_{i}+I_{j}, t\right)\right]+ \\
&+\sum_{\substack{c, s=1 \\
c, s \neq i}} \mu_{s}(t) \beta_{s c}\left(k+I_{c}-I_{s}, t\right) u\left(k_{s}\right) v_{i}\left(k+I_{c}-I_{s}, t\right)+ \\
&+\lambda(t) p_{0 i} \psi_{i j}\left(k+I_{i}, t\right) r_{0 i}\left(k+I_{i}, t\right)-\mu_{i}(t) \alpha_{i}\left(k-I_{i}, t\right) u\left(k_{i}\right) R_{i 0}\left(k-I_{i}, t\right)+r_{i}(k),
\end{aligned}
$$

\begin{tabular}{|c|c|c|}
\hline $\begin{array}{l}\text { Possible transitions } \\
\text { between network's } \\
\text { states }\end{array}$ & The transition probabilities & $\begin{array}{c}\text { Incomes of system } S_{i} \text { of } \\
\text { transitions between network's } \\
\text { states }\end{array}$ \\
\hline$(k, t) \rightarrow(k, t+\Delta t)$ & $\begin{aligned} & 1-\sum_{i=1}^{n}\left(\lambda(t) p_{0 i}\left(1-\varphi_{i}(k, t)\right)+\right. \\
+ & \left.\mu_{i}(t)\left(1-\beta_{i i}(k, t)\right)\right) \Delta t+o(\Delta t)\end{aligned}$ & $r_{i}(k) \Delta t+v_{i}(k, t)$ \\
\hline $\begin{array}{c}(k, t) \rightarrow\left(k+I_{j}, t+\Delta t\right), \\
j \neq i\end{array}$ & $\lambda(t) p_{0 j} \psi_{i j}\left(k+I_{j}, t\right) \Delta t+o(\Delta t)$ & $r_{i}(k) \Delta t+v_{i}\left(k+I_{j}, t\right)$ \\
\hline $\begin{array}{c}(k, t) \rightarrow\left(k-I_{j}, t+\Delta t\right) \\
j \neq i\end{array}$ & $\mu_{j}(t) \alpha_{j}\left(k-I_{j}, t\right) u\left(k_{j}\right) \Delta t+o(\Delta t)$ & $r_{i}(k) \Delta t+v_{i}\left(k-I_{j}, t\right)$ \\
\hline $\begin{array}{c}(k, t) \rightarrow \\
\left(k+I_{c}-I_{s}, t+\Delta t\right), \\
c, s \neq i\end{array}$ & $\mu_{s}(t) \beta_{s c}\left(k+I_{c}-I_{s}, t\right) u\left(k_{s}\right) \Delta t+o(\Delta t)$ & $r_{i}(k) \Delta t+v_{i}\left(k+I_{c}-I_{s}, t\right)$ \\
\hline$(k, t) \rightarrow\left(k+I_{i}, t+\Delta t\right)$ & $\lambda(t) p_{0 i} \psi_{i j}\left(k+I_{i}, t\right) \Delta t+o(\Delta t)$ & $r_{0 i}\left(k+I_{i}, t\right)+v_{i}\left(k+I_{i}, t\right)$ \\
\hline$(k, t) \rightarrow\left(k-I_{i}, t+\Delta t\right)$ & $\mu_{i}(t) \alpha_{i}\left(k-I_{i}, t\right) u\left(k_{i}\right) \Delta t+o(\Delta t)$ & $-R_{i 0}\left(k-I_{i}, t\right)+v_{i}\left(k-I_{i}, t\right)$ \\
\hline $\begin{array}{c}(k, t) \rightarrow \\
\left(k+I_{i}-I_{j}, t+\Delta t\right), \\
j \neq i\end{array}$ & $\mu_{j}(t) \beta_{j i}\left(k+I_{i}-I_{j}, t\right) u\left(k_{j}\right) \Delta t+o(\Delta t)$ & $\begin{array}{l}r_{i j}\left(k+I_{i}-I_{j}, t\right)+ \\
+v_{i}\left(k+I_{i}-I_{j}, t\right)\end{array}$ \\
\hline $\begin{array}{c}(k, t) \rightarrow \\
\left(k-I_{i}+I_{j}, t+\Delta t\right), \\
j \neq i\end{array}$ & $\mu_{i}(t) \beta_{i j}\left(k-I_{i}+I_{j}, t\right) u\left(k_{i}\right) \Delta t+o(\Delta t)$ & $\begin{array}{l}-r_{j i}\left(k-I_{i}+I_{j}, t\right)+ \\
+v_{i}\left(k-I_{i}+I_{j}, t\right)\end{array}$ \\
\hline
\end{tabular}

Table 1

Possible transitions between network's states, their probability and incomes of the system $S_{i}$ 
where $u(x)=\left\{\begin{array}{l}1, x>0 \\ 0, x \leq 0\end{array}\right.$ - the Heaviside function. The number of equations in this system is the number of states of the network. Relations for the conditional probabilities $\varphi_{i}(k, t), \psi_{i j}(k, t), \alpha_{i}(k, t), \beta_{i j}(k, t), i, j=\overline{1, n}$ are given at [1].

For closed networks, the system of equations (1) can be reduced to the final of the system of linear inhomogeneous ordinary differential equations (ODE) with constant coefficients, which in the matrix form can be written as

$$
\frac{d V_{i}(t)}{d t}=Q_{i}(t)+A(t) V_{i}(t)
$$

where: $V_{i}^{T}(t)=\left(v_{i}(1, t), v_{i}(2, t), \ldots, v_{i}(L, t)\right)$ - unknown vector of the system income $S_{i}, L$ - number of states of the network. The solution of (1) can be found by using the direct method, or the method of Laplace transforms. However, we should not forget that the number of states of a closed QN equals $L=C_{n+K-1}^{n-1}$, where: $K$ - number of messages served by the network, and it is quite large for a relatively small $n$ and $K$, i.e. the number of equations in (1) will also be large enough. Experience has shown that the method of Laplace transforms can make calculations for networks with relatively small state space $(L<300)$; the direct method can be carried out for networks of larger dimension than the method of Laplace transforms.

\section{Example 1}

Consider a closed network with the following parameters: $n=5, K=10$. Let intensity be $\lambda(t)=\lambda t, \mu_{i}(t)=\mu_{i}\left[\cos \left(\omega_{i} t\right)+1\right], i=\overline{1,5}$. The transition probabilities between the QS network are $p_{12}=p_{34}=p_{54}=1, p_{42}=p_{45}=0.3, p_{31}=p_{34}=p_{43}=$ $=0.4, p_{35}=0.2$. Because the network is closed, it equals the number of states $L=C_{n+K-1}^{n-1}=1001$. Write down some of them $(0,0,0,0,7),(0,0,0,1,6),(0,0,0,2,5)$, $(0,0,0,3.4),(0,0,0,4,3),(0,0,0,5,2),(0,0,0,6,1),(0,0,0,7,0),(0,0,1,0,6),(0,0,1,1,5)$ etc. Rename them from 1 to 1001 . Assume that the probabilities of the messages to join the queue at time $t$ is given by $f^{(i)}(t)=1-e^{-i t}, i=\overline{1,5}$. Conditional probabilities $\varphi_{i}(t), \psi_{i j}(t), \alpha_{i}(t)$ and $\beta_{i j}(t)$ are given at [1]: 


$$
\begin{gathered}
\varphi_{i}(k, t)=\left(1-f^{(i)}(k, t)\right)\left(p_{i 0}+\sum_{j=1}^{n} p_{i j} \varphi_{j}(k, t)\right), i=\overline{1, n}, \\
\psi_{i j}(k, t)=f^{(i)}(k, t) \delta_{i j}+\left(1-f^{(i)}(k, t)\right) \sum_{l=1}^{n} p_{i l} \psi_{l j}(k, t), i, j=\overline{1, n} \\
\alpha_{i}(k, t)=p_{i 0}+\sum_{j=1}^{n} p_{i j} \varphi_{j}\left(k-I_{i}, t\right), i=\overline{1, n}, \\
\beta_{i j}(k, t)=\sum_{l=1}^{n} p_{i l} \psi_{l j}\left(k-I_{i}, t\right), i, j=\overline{1, n}
\end{gathered}
$$

where $\delta_{i j}$ - the Kronecker delta. At [2] it was shown that they are cumbersome even at $n=3$. As the incomes of $r_{i}(k)$ were taken are some integers, $i=\overline{1.5}$. As incomes $r_{0 i}(l, t), R_{i 0}(l, t), r_{i j}(l, t), r_{j i}(l, t)$ were taken linear in $t$ not depending on states $k$ functions: $a t+b$, where: $a$ are some real numbers, and $b$ are some integers, $i, j=\overline{1.5}$, $i \neq j$. The initial conditions $V_{i}(0)$ - vector, consists of zeros, $i=\overline{1.5}$.

The numerical solution of the DDE (1) is obtained by using the mathematical calculations NDSolve package Mathematica, which by default is calculated with the help of the Runge-Kutta method of fourth order. The graph of system income $S_{3}$, on condition that at the initial moment network is in a state $(1,2,3,4,5)$ is shown at Figure 1.

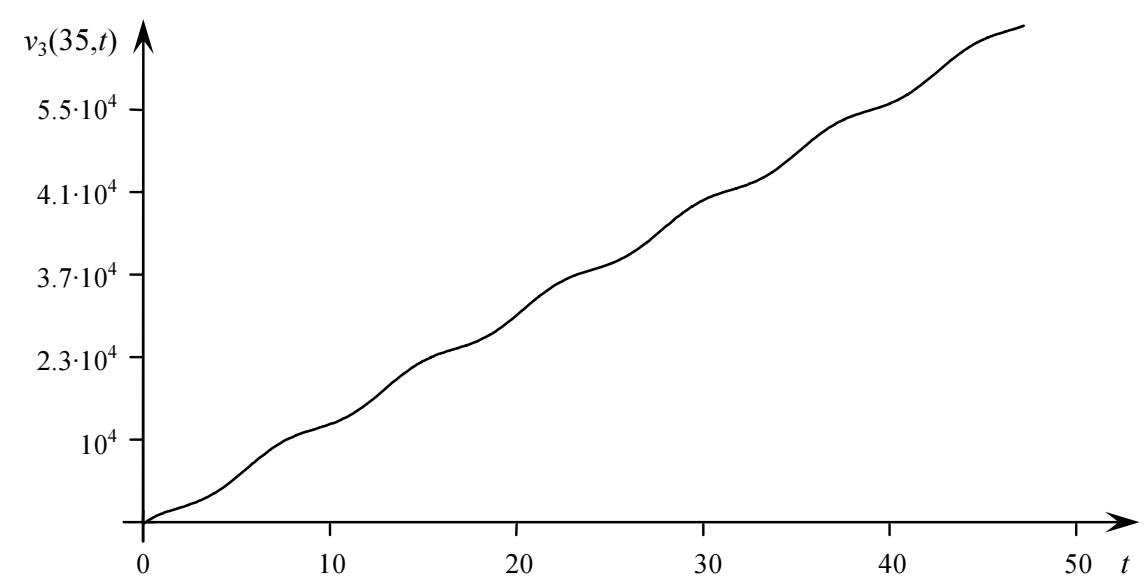

Fig. 1 . The graph of income change of system $S_{3}$ at the time interval $[0 ; 50]$ 


\section{Finding the expected incomes, when incomes from transitions between network states are functions that depend on the random variables}

Consider the case when the conditional probabilities $\varphi_{i}(k, t), \psi_{i j}(k, t), \alpha_{i}(k, t)$, $\beta_{i j}(k, t)$, do not depend on the state of the network, i.e. $\varphi_{i}(k, t)=\varphi_{i}(t)$, $\psi_{i j}(k, t)=\psi_{i j}(t), \alpha_{i}(k, t)=\alpha_{i}(t), \beta_{i j}(k, t)=\beta_{i j}(t), i, j=\overline{1, n}$. As before, we consider the case when the revenue from the state transition networks are RV or functions that depend on them [3-5]. Let RV $\xi_{i}$ - service time messages in the system $S_{i}$, distributed exponentially with the distribution function (DF) $F_{\xi_{i}}(t)=1-e^{-\mu_{i}(t) t}$, $i=\overline{1, n}$. Consider the dynamic of system $S_{i}$ income Suppose that at the initial moment income of the system is equal to $v_{i 0}$. We are interested in income of the system $V_{i}(t)$ at time moment $t$. We subdivide the interval $[0, t]$ into $m$ equal parts of length $\Delta t=\frac{t}{m}$, considering the $m$ large enough to find the income the system $S_{i}$ write out the conditional probabilities of the events that may occur in the $l$-th time interval, $l=\overline{1, m}$. All possible transitions between network states, their probabilities and incomes of system $S_{i}$ from such transitions are following:

1. With the probability $p_{i}^{(1)}(l, t, \Delta t)=\lambda(t) p_{0 i} \psi_{i j}(t) \Delta t+o(\Delta t)$ the system $S_{i}$ received message from the external environment that will bring it an in come of $r_{0 i}$, where: $r_{0 i}$ - RV with the expectation (m.e.) $M\left(r_{0 i}\right)=a_{0 i}, i=\overline{1, n}$.

2. With the probability $p_{i}^{(2)}(l, t, \Delta t)=\mu_{i}(t) \varepsilon_{i}^{(l)} \alpha_{i}(t) u\left(k_{i}^{(l)}\right) \Delta t+o(\Delta t)$ message from the system $S_{i}$ enters into the environment, with the income of the system $S_{i}$ reduced by the value $R_{i 0}$, where: $R_{i 0}$ - RV with m.e. $M\left(R_{i 0}\right)=b_{i 0}, k_{i}^{(l)}$ - the number of messages in the system $S_{i}$ (in a queue and serviced) on $l$-th time interval, $l=\overline{1, m}, i=\overline{1, n}$. Value $\varepsilon_{i}^{(l)}$ means the number of lines of messages for employment $l$-th time interval in the system $S_{i}, i=\overline{1, n}$.

3. The message from the system $S_{i}$ transformed into the system $S_{j}$ with the probability $p_{i j}^{(3)}(l, t, \Delta t)=\mu_{i}(t) \varepsilon_{i}^{(l)} u\left(k_{i}^{(l)}\right) \beta_{i j}(t) \Delta t+o(\Delta t), j=\overline{1, n}, i \neq j$. Such a transition gives the system $S_{i}$ income reduced by the value $R_{i j}\left(\xi_{i}\right)$, and the income of the system $S_{j}$ increased by this value, in which $R_{i j}\left(\xi_{i}\right)$ - the casual income (loss) of the system $S_{i}$ because of the transition from the message the system $S_{i}$ 
into system $S_{j}, \quad M\left\{R_{i j}\left(\xi_{i}\right)\right\}=\int_{0}^{\infty} R_{i j}(t) d F_{\xi_{i}}(t)=\int_{0}^{\infty} R_{i j}(t) e^{-\mu_{i}(t) t} d t=\gamma_{i j}, \quad i=\overline{1, n}$, $j=\overline{1, n}, i \neq j$.

4. With the probability

$$
\begin{aligned}
p^{(4)}(l, t, \Delta t)=1 & -\left(\lambda(t) \sum_{i=1}^{n} p_{0 i} \sum_{\substack{j=1 \\
j \neq i}}^{n} \psi_{i j}(t)+\sum_{i=1}^{n} \mu_{i}(t) \alpha_{i}(t) \varepsilon_{i}^{(l)} u\left(k_{i}^{(l)}\right)-\right. \\
& +\sum_{i=1}^{n} \mu_{i}(t) \varepsilon_{i}^{(l)} u\left(k_{i}^{(l)}\right) \sum_{j=1}^{n} \beta_{i j}(t)- \\
- & \left.\sum_{i=1}^{n}\left[\lambda(t) p_{0 i}\left(1-\varphi_{i}(t)\right)+\mu_{i}(t)\left(1-\beta_{i i}(t)\right)\right]\right) \Delta t+o(\Delta t)
\end{aligned}
$$

at the time interval $\Delta t$ network status will not change.

In addition, for each small time interval $\Delta t$ system $S_{i}$ because of containing a message, increases the value of the income $\eta_{i} \Delta t$, where $\eta_{i}-\mathrm{RV}$ with m.e. $M\left\{\eta_{i}\right\}=\bar{\eta}_{i}, i=\overline{1, n}$. We also assume that $\mathrm{RV} r_{0 i}, R_{0 i}, R_{i j}\left(\xi_{i}\right)$ are pairwise independent, $i=\overline{1, n}, j=\overline{1, n}$.

Let $\Delta V_{i l}(\Delta t)$ - changes in the income of system $S_{i}$ on $l$-th time interval associated with the transitions between the QS network applications. Then it follows from the above:

$$
\Delta V_{i l}(\Delta t)=\left\{\begin{array}{l}
r_{0 i}+\eta_{i} \Delta t \text { with the probability } p_{i}^{(1)}(l, t, \Delta t), \\
-R_{i 0}+\eta_{i} \Delta t \text { with the probability } p_{i}^{(2)}(l, t, \Delta t), \\
-R_{i j}\left(\xi_{i}\right)+\eta_{i} \Delta t \text { with the probability } p_{i j}^{(3)}(l, t, \Delta t), j=\overline{1, n}, j \neq i, \\
\eta_{i} \Delta t \text { with the probability } p^{(6)}(l, t, \Delta t) .
\end{array}\right.
$$

The total income of the system $S_{i}$ is equal to

$$
V_{i}(t)=v_{i 0}+\sum_{l=1}^{m} \Delta V_{i l}(\Delta t)=v_{i 0}+V_{i}(\Delta t)
$$

where $V_{i}(t)=\sum_{l=1}^{m} \Delta V_{i l}(\Delta t)$ - income of the system $S_{i}$ from moving messages, $i=\overline{1, n}$.

We find an expression for the expected income of the system $S_{i}$ at time $t$. Using the formula of total probability for the expectation, for a fixed realization of the process $k(t)$ we can write: 


$$
\begin{gathered}
M\left\{\Delta V_{i l}(\Delta t) / k(t)\right\}=\left(a_{0 i}+\bar{\eta}_{i} \Delta t\right) p_{i}^{(1)}(l, t, \Delta t)+\left(-b_{i 0}+\bar{\eta}_{i} \Delta t\right) p_{i}^{(2)}(l, t, \Delta t)+ \\
+\left(-\gamma_{i j}+\bar{\eta}_{i} \Delta t\right) p_{i j}^{(3)}(l, t, \Delta t)+\bar{\eta}_{i} p^{(4)}(l, t, \Delta t) \Delta t, i=\overline{1, n}
\end{gathered}
$$

Further, substituting functions $p_{i}^{(1)}(l, t, \Delta t), p_{i}^{(2)}(l, t, \Delta t), p_{i j}^{(3)}(l, t, \Delta t)$ and $p^{(4)}(l, t, \Delta t)$ for suitable transition probabilities, we obtain

$$
\begin{gathered}
M\left\{\Delta V_{i l}(\Delta t) / k(t)\right\}=\left(a_{0 i}+\bar{\eta}_{i} \Delta t\right)\left(\lambda(t) p_{0 i} \sum_{j=1}^{n} \psi_{i j}(t) \Delta t+o(\Delta t)\right)+ \\
+\left(-b_{i 0}+\bar{\eta}_{i} \Delta t\right)\left(\mu_{i}(t) \alpha_{i}(t) \varepsilon_{i}^{(l)} u\left(k_{i}^{(l)}\right) \Delta t+o(\Delta t)\right)+ \\
+\left(-\sum_{j=1}^{n} \gamma_{i j}+\bar{\eta}_{i} \Delta t\right)\left(\mu_{i}(t) \varepsilon_{i}^{(l)} u\left(k_{i}^{(l)}\right) \sum_{j=1}^{n} \beta_{i j}(t) \Delta t+o(\Delta t)\right)+ \\
+\bar{\eta}_{i} \Delta t\left[1-\left(\lambda(t) \sum_{i=1}^{n} \sum_{c=1}^{r} p_{0 i} \sum_{\substack{j=1 \\
j \neq i}}^{n} \psi_{i j}(t)+\sum_{i=1}^{n} \mu_{i}(t) \alpha_{i}(t) \varepsilon_{i}^{(l)} u\left(k_{i}^{(l)}\right)+\sum_{i=1}^{n} \mu_{c}(t) \varepsilon_{i}^{(l)} u\left(k_{i}^{(l)}\right) \sum_{j=1}^{n} \beta_{i j}(t)-\right.\right. \\
\left.\left.-\sum_{i=1}^{n}\left[\lambda(t) p_{0 i}\left(1-\varphi_{i}(t)\right)+\mu_{i}(t)\left(1-\beta_{i i}(t)\right)\right]\right) \Delta t+o(\Delta t)\right], i=\overline{1, n}
\end{gathered}
$$

Then, given that $m \Delta t=t$, expanding and making some transformations it can be written:

$$
\begin{gathered}
M\left\{V_{i}(t) / k(t)\right\}=\sum_{l=1}^{m} M\left\{\Delta V_{i l}(\Delta t) / k(t)\right\}= \\
=\sum_{i=1}^{n-1}\left[a_{0 i} \lambda(t) p_{0 i} \sum_{j=1}^{n} \psi_{i j}(t)-\mu_{i}(t) \varepsilon_{i}^{(l)} u\left(k_{i}^{(l)}\right)\left(b_{i 0} \alpha_{i}(t)+\sum_{j=1}^{n} \beta_{i j}(t) \gamma_{i j}\right)+\bar{\eta}_{i}\right] t- \\
-m \sum_{i=1}^{n-1} \bar{\eta}_{i}\left[\sum_{i=2}^{n}\left(\lambda(t) p_{0 i} \sum_{j=1}^{n} \psi_{i j}(t)+\mu_{i}(t) \varepsilon_{i}^{(l)} u\left(k_{i}^{(l)}\right)\left(\alpha_{i}(t)+\sum_{j=1}^{n} \beta_{i j}(t)\right)\right]-\right. \\
\left.-\sum_{i=1}^{n}\left[\lambda(t) p_{0 i}\left(1-\varphi_{i}(t)\right)+\mu_{i}(t)\left(1-\beta_{i i}(t)\right)\right]\right] \Delta t+o(\Delta t), i=\overline{1, n}
\end{gathered}
$$

Obtain expressions for the expected incomes $v_{i}(t)=M\left\{V_{i}(t)\right\}$. Averaging over $k(t)$ with the normalization condition $\sum_{k} P(k(t)=k)=1$, for the expected income of the system $S_{i}$ we have 


$$
\begin{gathered}
v_{i}(t)=M\left\{V_{i}(t)\right\}=\sum_{k} P(k(t)=k) M\left\{V_{i}(t) / k(t)\right\}= \\
=\left[a_{0 i} \lambda(t) p_{0 i} \sum_{j=1}^{n} \psi_{i j}(t)-\mu_{i}(t) \sum_{l=1}^{m} \varepsilon_{i}^{(l)} u\left(k_{i}^{(l)}\right)\left(b_{i 0} \alpha_{c}(t)+\sum_{j=1}^{n} \beta_{i j}(t) \gamma_{i j}\right)+\bar{\eta}_{i}\right] t- \\
-\sum_{k} P(k(t)=k) \bar{\eta}_{i}\left[\sum_{i=2}^{n}\left(\lambda(t) p_{0 i} \sum_{j=1}^{n} \int_{0}^{t} \psi_{i j}(x) d x+\mu_{i}(t)\left(\alpha_{i}(t)+\sum_{j=1}^{n} \beta_{i j}(t)\right) \sum_{l=1}^{m} \varepsilon_{i}^{(l)} u\left(k_{i}^{(l)}\right) \Delta t\right)-\right. \\
-\sum_{i=1}^{n}\left[\lambda(t) p_{0 i}\left(1-\varphi_{i}(t)\right)+\mu_{i}(t)\left(1-\beta_{i i}(t)\right)\right], i=\overline{1, n} .
\end{gathered}
$$

We denote the expectation

$$
M\left\{\varepsilon_{i}(t) u\left(k_{i}(t)\right)\right\}=\rho_{i}(t), i=\overline{1, n},
$$

where $\rho_{i}(t)$ - the average number of employed service lines in the system $S_{i}$ messages at the time moment $t, i=\overline{1, n}$.

At $m \rightarrow \infty$ it will be $\Delta t \rightarrow 0$ and:

$$
\sum_{l=1}^{m} \varepsilon_{i}^{(l)} u\left(k_{i}^{(l)}\right) \Delta t \underset{\Delta t \rightarrow 0}{\longrightarrow} \int_{0}^{t} \varepsilon_{i}(x) u\left(k_{i}(x)\right) d x, i=\overline{1, n},
$$

where: $k_{i}(x)$ - the number of messages in system $S_{i}$ at the time moment $x, \varepsilon_{i}(x)$ number of employed lines in the system messages $S_{i}$ at the time moment $x$.

According to (10) and (11), we obtain the following approximate relationship:

$$
\begin{gathered}
v_{i}(t)=M\left\{V_{i}(t)\right\}=v_{i 0}+v_{i}(t)=v_{i 0}+ \\
+\left[a_{0 i} \lambda(t) p_{0 i} \sum_{j=1}^{n} \psi_{i j}(t)-\mu_{i}(t)\left(b_{i 0} \alpha_{i}(t)+\sum_{j=1}^{n} \beta_{i j}(t) \gamma_{i j} \int_{0}^{t} \rho_{i}(x) d x+\bar{\eta}_{i}\right] t-\right. \\
-\bar{\eta}\left[\sum_{i=2}^{n}\left(p_{0 i} \sum_{j=1}^{n} \lambda(t) \psi_{i j}(t)+\left(\alpha_{i}(t)+\sum_{j=1}^{n} \beta_{i j}(t)\right) \mu_{i}(t) \int_{0}^{t} \rho_{i}(x) d x\right)-\right. \\
-\sum_{i=1}^{n}\left[p_{0 i}\left(\lambda(t)-\lambda(t) \varphi_{i}(t)\right)+\mu_{i}(t)-\mu_{i}(t) \beta_{i i}(t)\right], i=\overline{1, n}
\end{gathered}
$$

To find the value of $\rho_{i}(t)$ you can use the technique, finding the average number of occupied lines in network systems, as described in [4]. 
Consider the special case when we have the HM-network without messages in [4] the open Multiline HM-network with heterogeneous messages. Mesages in the transition between systems network can change its type. Consider the case when the incomes from the state transition network are RV or functions that depend on them. Expressions were obtained for the expected incomes systems for each type of network messages. In the case of similar messages, the expressions for the expected incomes systems in the network, obtained in [4], took the form

$$
\begin{gathered}
v_{i}(t)=v_{i 0}+\left[a_{0 i}\left(1+\lambda(t)\left(p_{0 i}-\sum_{i=1}^{n} p_{0 i}\right) \int_{0}^{t} \rho_{i}(x) d x\right)+\bar{\eta}\right] t- \\
-\bar{\eta} \mu_{i}(t)\left(b_{i 0} p_{i 0}-\sum_{j=1}^{n} p_{i j} \gamma_{i j}\right) \int_{0}^{t} \rho_{i}(x) d x+\bar{\eta} \sum_{i=1}^{n} \sum_{j=1}^{n} \mu_{i}(t) p_{i j} \int_{0}^{t} \rho_{i}(x) d x, i=\overline{1, n} .
\end{gathered}
$$

In our case, $f^{(i)}(k, t)=1$ then the expression for the conditional probabilities $\varphi_{i}(t), \psi_{i j}(t), \alpha_{i}(t)$ and $\beta_{i j}(t)$, According to [1], there are as follows:

$$
\begin{gathered}
\varphi_{i}(t)=\left(1-f^{(i)}(t)\right)\left(p_{i 0}+\sum_{j=1}^{n} p_{i j} \varphi_{j}(t)\right)=0, i=\overline{1, n}, \\
\psi_{i j}(t)=f^{(i)}(k, t) \delta_{i j}+\left(1-f^{(i)}(t)\right) \sum_{l=1}^{n} p_{i l} \psi_{l j}(t)=\delta_{i j}, i, j=\overline{1, n}, \\
\alpha_{i}(t)=p_{i 0}+\sum_{j=1}^{n} p_{i j} \varphi_{j}(t)=p_{i 0}, i=\overline{1, n}, \\
\beta_{i j}(t)=\sum_{l=1}^{n} p_{i l} \psi_{l j}(t)=\sum_{l=1}^{n} \delta_{l j}(t) p_{i l}, i, j=\overline{1, n} .
\end{gathered}
$$

Substituting them into (8), we obtain the expression (9). This confirms the correctness of the research and the results on an open HM-network with the same type messages bypass of systems in this case.

\section{Example 2}

Consider an open network $n=5, K=100$, other parameters as in Example 1 . Let us leave all the initial data the same as in the Example 1. Let the service rates be equal $\mu_{i}(t)=\frac{\mu_{i}}{t}, i=\overline{1.5}$. Vector of the QS network looks like $m=\left(m_{1}, m_{2}, \ldots, m_{n}\right)=$ $=(10,30,7,1,10)$. Random incomes systems are: $R_{i j}\left(\xi_{i}\right)=3000 \xi_{i}, i, j=\overline{1.5}$. Con- 
sider the time period of 24 hours, $t \in[0, T], T=24$. Mathematical expectations $M\left\{\eta_{i}\right\}=\bar{\eta}_{i}$, and $M\left\{R_{i j}\left(\xi_{i}\right)\right\}=\gamma_{i j}, i, j=\overline{1, n}$, are equals respectively: $\bar{\eta}_{1}=100$, $\bar{\eta}_{2}=200, \bar{\eta}_{3}=\bar{\eta}_{4}=\bar{\eta}_{5}=500, \gamma_{i j}=3000 \int_{0}^{24} t e^{-\mu_{i}} d t=864000 e^{-\mu_{i}}$.

Let at the initial moment $t_{0}=0$ incomes system be zero. Then, using the formula (7) and the package of mathematical calculations Wolfram Mathematica, expressions were obtained for the changes expected in the incomes systems $S_{i}$ $i=\overline{1, n}$, and the income of the whole network at the time interval. The graph of this function is shown at Figure 2, and the graph of the expected income of the whole network - in Figure 3.

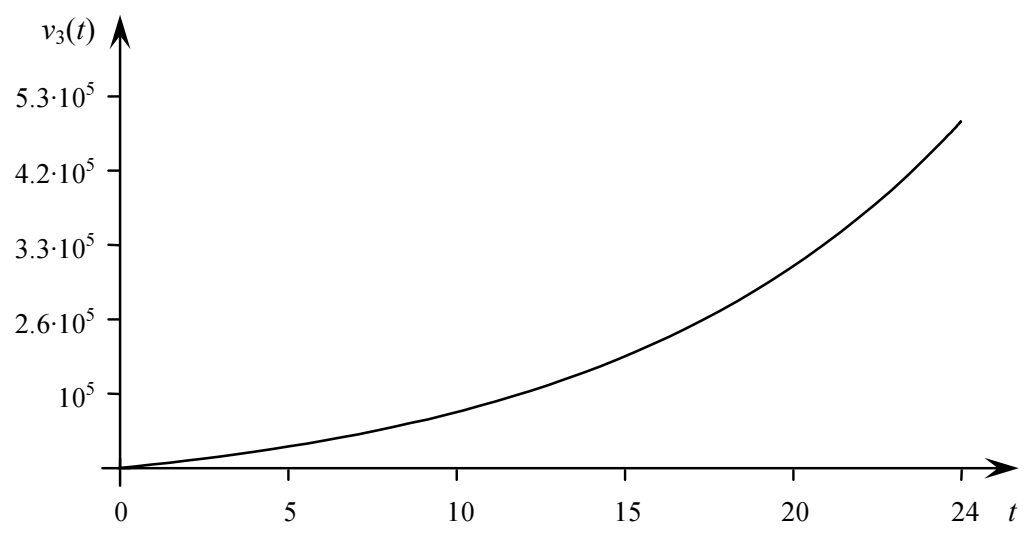

Fig. 2. The graph of change of expected income in the system $S_{3}$

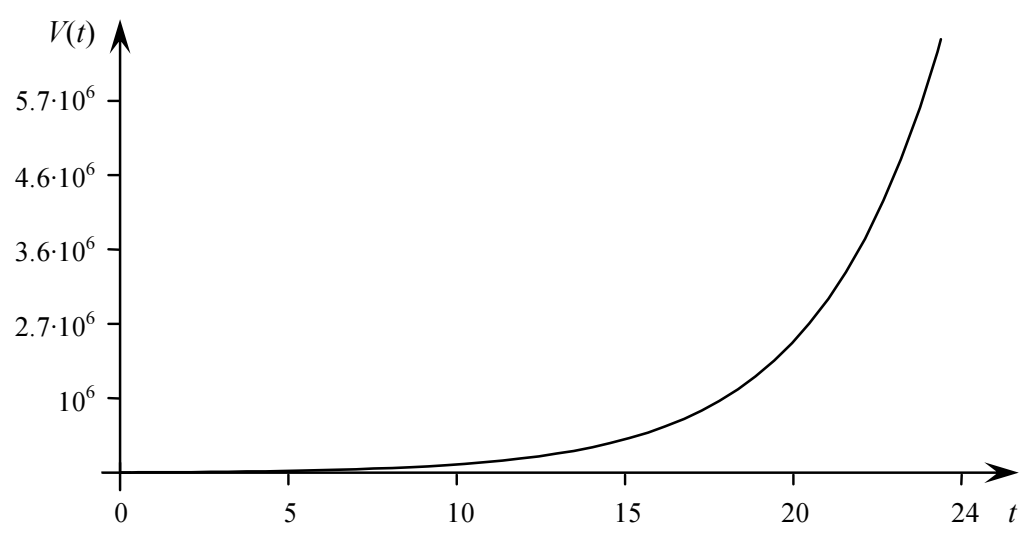

Fig. 3. The graph of change of expected income of the whole network 


\section{Conclusions}

An open HM-network with one-type messages bypass of systems in the transient behaviour was investigated. Two cases were considered: when incomes from transitions between network states are deterministic functions depending on states and time, and network systems are single-line, and when incomes from transitions between network states are functions depending on the random variables.

The expected incomes of such a network were found in both cases on condition that the probabilities messages bypasses of systems network, parameters of incoming flow of messages and services depend on time. Two examples were considered and calculated.

\section{References}

[1] Matalytski M., Naumenko V., Analysis of the queuing network with messages bypass of systems in transient behavior, Scientific Research of the Institute of Mathematics and Computer Science 2012, 11(2), 71-83.

[2] Matalytski M., Naumenko V., Analysis of networks with time-dependent transition probabilities and messages bypass between the queuing systems, Scientific Research of the Institute of Mathematics and Computer Science, 2012, 11(2).

[3] Naumenko V., Model of incomes forecasting points shared information network, Proceedings of the International Congress on Computer Science: Information Systems and Technologies, 2011, 41-46 (in Russian).

[4] Naumenko V., Investigation of the model of changes incomes at item of collective use, Vestnik GrSU, 2012, 2, 143-157 (in Russian). 
